Compact MEMS-based Adaptive Optics Optical Coherence Tomography for Clinical Use

D. Chen, S. Olivier, S. Jones, R. Zawadzki, J. Evans, S. Choi, J. Werner

February 27, 2008

SPIE Photonics West 2008

San Jose, United States

January 18, 2008 through January 24, 2008 
This document was prepared as an account of work sponsored by an agency of the United States government. Neither the United States government nor Lawrence Livermore National Security, LLC, nor any of their employees makes any warranty, expressed or implied, or assumes any legal liability or responsibility for the accuracy, completeness, or usefulness of any information, apparatus, product, or process disclosed, or represents that its use would not infringe privately owned rights. Reference herein to any specific commercial product, process, or service by trade name, trademark, manufacturer, or otherwise does not necessarily constitute or imply its endorsement, recommendation, or favoring by the United States government or Lawrence Livermore National Security, LLC. The views and opinions of authors expressed herein do not necessarily state or reflect those of the United States government or Lawrence Livermore National Security, LLC, and shall not be used for advertising or product endorsement purposes. 


\title{
Compact MEMS-based Adaptive Optics Optical Coherence Tomography for Clinical Use
}

\author{
Diana Chen ${ }^{1}$, Scot Olivier ${ }^{1}$, Steven Jones ${ }^{1}$, \\ Robert Zawadzki ${ }^{2}$, Julie Evans ${ }^{2}$, Stacey Choi ${ }^{2}$, and Jack Werner ${ }^{2}$ \\ ${ }^{1}$ Lawrence Livermore National Laboratory, \\ 6000 East Avenue, Livermore, CA 94550 \\ ${ }^{2}$ Department of Ophthalmology and Vision Science, \\ UC Davis, 4860 Y Street, Suite 2400
}

\begin{abstract}
We describe a compact MEMS-based adaptive optics (AO) optical coherence tomography system with improved AO performance and ease of clinical use. A typical AO system consists of a Shack-Hartmann wavefront sensor and a deformable mirror that measures and corrects the ocular and system aberrations. Because of the limitation on the current deformable mirror technologies, the amount of real-time ocular-aberration compensation is restricted and small in the previous AO-OCT instruments. In this instrument, we proposed to add an optical apparatus to correct the spectacle aberrations of the patients such as myopia, hyperopia and astigmatism. This eliminated the tedious process of the trial lenses in clinical imaging. Different amount of spectacle aberration compensation was achieved by motorized stages and automated with the AO computer for ease of clinical use. In addition, the compact AO-OCT was optimized to have minimum system aberrations to reduce $\mathrm{AO}$ registration errors and improve $\mathrm{AO}$ performance.
\end{abstract}

Keywords: Adaptive optics, ophthalmic optics, optical coherent tomography, eye, retina

\section{INTRODUCTION}

Optical coherent tomography (OCT) is a leading modality for non-invasive, in vivo imaging of the human retina, providing high sensitivity and axial resolution in quantifying visual function and providing retinal pathology of the individual cells for the retina. The lateral resolution of the OCT image of the eye, however, is poor due to ocular aberrations.

Adaptive optics (AO) has been incoprated into OCT system to increase the lateral resolution by measuring and subsequent compensating the aberrations in real time. The technology was originally used for correcting the image degradation due to atmospheric aberration in astronomy. In recent years, AO technology has been applied to the imaging instruments for eyes, such as flood illumination fundus imaging ${ }^{[1]}$, scanning laser ophthalmoscopy $^{[2]}$, and most recently ophthalmic optical coherent tomography ${ }^{[3]}$. In an AO system, the ocular aberrations of the test subjects are measured by a wavefront 
sensor. The measured wavefront errors are then used to adjust the shape of a deformable mirror (DM) until the wavefront aberrations are minimized.

Population studies have shown that many people have both low-order aberrations with large magnitudes and high-order aberrations with small magnitudes. For these subjects, current technology cannot deliver the phase compensation needed using a single DM. The use of two deformable mirrors has been investigated.

The first AO-OCT system incorporating two deformable mirrors was demonstrated by Zawadzki et.al ${ }^{[4]}$. The bimorph DM from AOptix used in UC Davis OCT system had a relatively high dynamic range and could correct defocus and astigmatism up to $\pm 3 \mathrm{D}$. This obviated the need for trial lenses and the meticulous use of such lenses to correct individual subject's refractive errors. The MEMS from Boston Micro Machine had 144 pixels and $1.5 \mu \mathrm{m}$ stroke, and was used to correct the residual high-order aberrations left by the biomorph DM compensation. Both deformable mirrors were placed in the nonscanning path. Such arrangement, however, generated noticeable beam distortions at the deformable mirrors and the wavefront sensor when large refractive corrections are needed ${ }^{[5]}$.

In the AO-OCT system demonstrated by Zhang et al, the bimorph mirror was placed one relay telescope away from the eye. This minimized the propagation of ocular refractive errors through the system prior to compensation. This arrangement greatly reduced the pupil distortion at the deformable mirrors and wavefront sensor. However, because the bimorph mirror was placed in the scanning path between the eye and scanners, the beam at the pupil of the patient shifted with the changing incident angles of the light as the beam steered by the scanners. This would result in degradation of the AO-OCT system.

To address these issues, we added an optical apparatus to correct the spectacle aberrations of the patients such as myopia, hyperopia and astigmatism. The residual aberrations were compensated by MEMS, which was placed between the SLD light source and XY scanners in the AO-OCT system so that the pupil shift at the MEMS was minimized. In addition, the compact AO-OCT was optimized to have minimum system aberrations to reduce $\mathrm{AO}$ registration errors and improve $\mathrm{AO}$ performance. Unlike the previous large systems which were set-up on a standard lab table, the proposed AO-OCT system was specially designed for clinical use requiring compact size, low cost and high reliability.

\section{OPTICAL SYSTEM}

The AO-OCT system consists of three channels: (1) sample channel, equipped with AO for collection the retinal image, (2) reference channel with an optical path length, matching that of the sample channel, and (3) detection channel for recording the combined sample and reference signals. 
The major components of the system are summarized in the following table:

\begin{tabular}{|c|c|}
\hline Light source & $\begin{array}{l}\text { Superluminescent diode (SLD) from Superlum } \\
\lambda=842 \mathrm{~nm}, \delta \lambda=50 \mathrm{~nm}\end{array}$ \\
\hline Deformable mirror & $\begin{array}{l}\text { MEMS from Boston Michomachines Corp. } \\
3.3 \mathrm{~mm} \text { x } 3.3 \mathrm{~mm} \text { optical aperture, } 12 \times 12 \text { actuators } \\
1.5 \mu \mathrm{m} \text { stroke }\end{array}$ \\
\hline $\begin{array}{l}\text { Horizontal/vertical } \\
\text { scanners }\end{array}$ & $\begin{array}{l}\text { Cambridge Technology; 6220M40 galvanometric } \\
\text { scanner, } \pm 20^{\circ}\end{array}$ \\
\hline Wavefront sensor & $\begin{array}{l}\text { Shack-hartmann wavefront sensor } \\
\text { Lens array from Adaptive Optics Associates } \\
\text { 20x20 elements, } 500 \mu \mathrm{m} \text { pitch, } 30 \mathrm{~mm} \text { focal length } \\
\text { CCD camera from Dalsa }\end{array}$ \\
\hline Diffraction grating & $\begin{array}{l}\text { Holographic transmitting diffraction grating from Wasatch } \\
\text { Photonics } \\
\text { 1200line } / \mathrm{mm}\end{array}$ \\
\hline CCD camera & $\begin{array}{l}\text { Line-scan camera from Atmel, } \\
12 \text { bit, } 2048 \text { pixels }\end{array}$ \\
\hline
\end{tabular}

In the sample channel, the light from a broadband superluminescent diode (SLD) was coupled into a single mode fiber. The light was then collimated and relayed by mirror telescopes to the deformable mirror, the horizontal and vertical scanners, the sphericalcylindrical correction apparatus and finally to the eye. Each component was placed at the image plane of an afocal relay telescope. Spherical mirrors, instead of lenses, were used in the afocal telescope designs to reduce back-reflections and minimize chromatic aberrations in the system. This is very important because the spectrum of the light source must be very broad to achieve high axial resolution. The off-axis configuration of the reflective spherical mirrors, however, created substantial system aberrations. It also resulted in beam displacement at the pupil of the eye due to scanning in both X-Y directions. Both aberrations and beam displacements resulted in performance degradation of the AO-OCT system. The sample channel was very different from the conventional OCT in the sense that it could measure the ocular aberrations of the patients and compensate aberrations in real-time to achieve the substantial higher lateral resolution. We will discuss the optical design issues and new configurations in details.

Reference channel and detection channel were similar to the conventional OCT. In the reference channel, the light was bouncing back and forth by several spherical mirrors. The optical path length must match that of the sample channel. In the detection channel, the light from the sample and reference arms was split by the fiber coupler and sent to the detector which was basically a spectrometer. At the input, the light was collimated to a holographic transmitting diffraction grating then focused onto a line-scan CCD. 


\section{OPTICAL SYSTEM ABERRATIONS:}

The off-axis configuration of the reflective spherical mirrors created substantial system aberrations $^{[6]}$. Theoretically, the deformable mirror should be able to compensate both optical system aberrations as well as the ocular aberrations from the patients. However, if part of the strokes of the deformable mirror was used to compensate the system aberrations, the magnitude of the compensation for the ocular aberrations was reduced. Furthermore, the aberrations would introduce pupil aberration and distortion, which would cause performance degradation the AO compensation. Thus the aberrations from the optical system itself should be minimized.

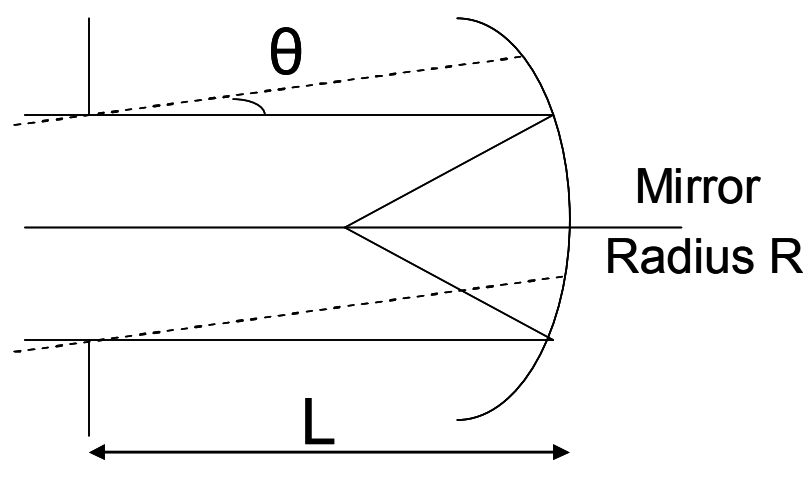

Fig 1. Illustration of a spherical mirror with a radius of curvature $\mathrm{R}$. The entrance pupil is at distance $\mathrm{L}$ from the vertex of the mirror. Incident angle of the beam is $\theta$.

For a single reflective spherical mirror such as the one shown in Fig. 1, the magnitude of aberrations is given in the second column

\begin{tabular}{|l|l|l|}
\hline Aberrations & Magnitude & When $L=R / 2$ \\
\hline Spherical aberration & $\frac{1}{128 F^{3}}$ & $\frac{1}{128 F^{3}}$ \\
\hline Coma & $\frac{(L-R) \theta)}{16 R F^{2}}$ & $\frac{\theta}{32 F^{2}}$ \\
\hline Astigmatism & $\frac{(L-R)^{2} \theta^{2}}{2 R^{2} F}$ & $\frac{\theta^{2}}{8 F}$ \\
\hline
\end{tabular}

Where the radius of curvature is $\mathrm{R}$ and incident angle is $\theta$. The entrance pupil is at distance $\mathrm{L}$ from the vertex of the mirror. F stands for the F-number. 
In a set of afocal telescope, $\mathrm{L}$ is half of $\mathrm{R}$. The magnitude of the aberrations is simplified in the third column. Examination of the magnitude in details provides important guidelines to the optical design.

1) Spherical/Coma/Astigmatism $\sim 1 / \mathrm{F}^{3}: 1 / \mathrm{F}^{2}: 1 / \mathrm{F}$

Aberrations decrease as the F-number increases. So afocal telescopes with longer focal lengths have smaller aberrations

2) Spherical/Coma/Astigmatism $\sim 1: \theta: \theta^{2}$

Spherical aberration is independent of the tilt angle of the reflective mirror. Both coma and astigmatism increases as the tilt angle increases, so afocal telescopes with smaller tilt angle has smaller aberrations

3) Spherical/Coma/Astigmatism $=1: 4 \theta \mathrm{F}:(4 \theta \mathrm{F})^{2}$

For a mirror with a radius of curvature of $0.5 \mathrm{~m}$, entrance pupil diameter of $10 \mathrm{~mm}$ and tilt angle of $5^{\circ}$, the ratio of Spherical/Coma/Astigmatism=1/8.7/75.7. In most cases, astigmatism is the dominating aberration in the system

In addition, most of telescopes typically form images over a curved surface instead of a flat surface. The aberration of the field curvature can be expressed as a defocus term $\theta^{2}$ $/ 16 \mathrm{R}_{\mathrm{p}} \mathrm{F}^{2}$ where $\mathrm{R}_{\mathrm{p}}$ is the radius of Petzval field curvature. A system with small tilt angle and large focal length thus has smaller aberration due to field curvature.

\section{Design implementation:}

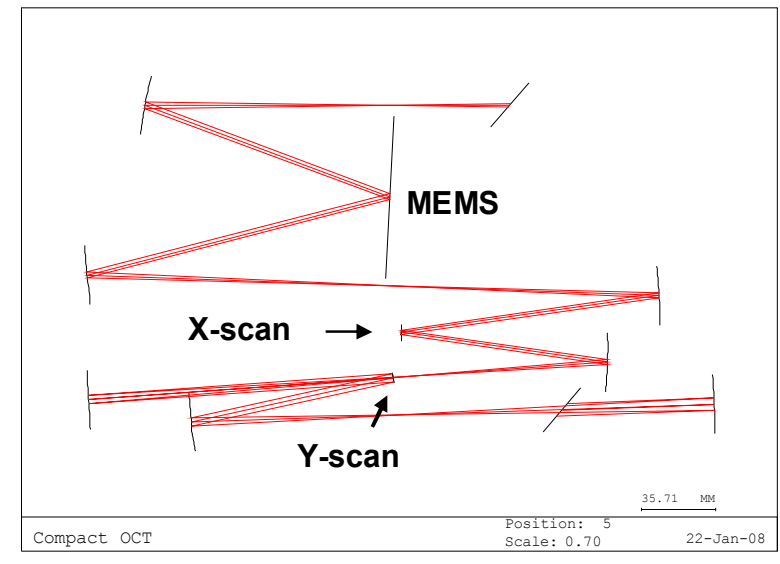

Fig. 2(a)

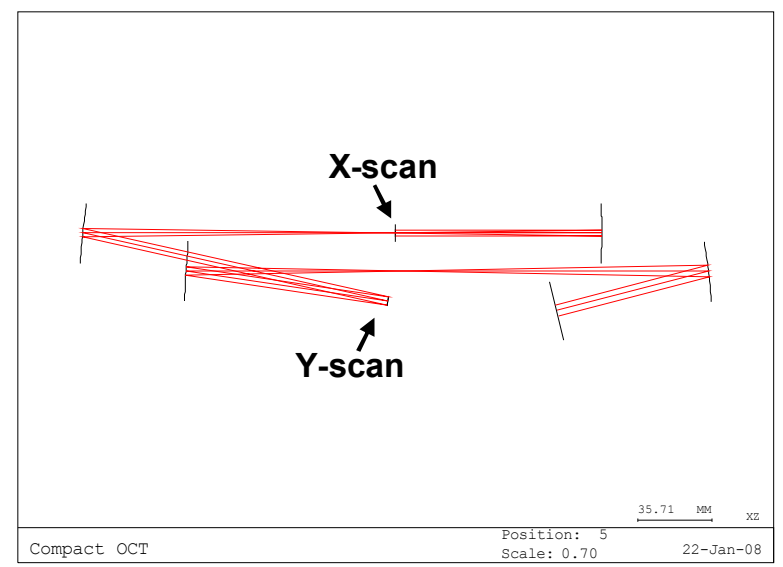

Fig. 2(b)

Fig 2. The optical design of an AO-OCT sample channel. The two mirrors in the relay telescope between horizontal scanner and vertical scanner were tilted orthogonally. (a) Optical layout in YZ plane (b) Optical layout in XZ plane. 
The above analysis presents the challenges for the design of a compact AO-OCT system. Long focal length results in large footprint. Small tilt angle would cause physical conflicts between the components. We minimized the total aberrations by targeting astigmatism because it is the dominating aberration in the afocal telescope. By tilting the second spherical mirror of the afocal telescope in the orthogonal plane, the two spherical mirrors cancelled the astigmatism introduced by a single mirror. The additional benefit was that the mirrors were now at different heights, so the physical conflict was less an issue for the smaller tilt angles.

Fig. 2 showed the design layout of an AO-OCT sample channel. The two mirrors in the relay telescope between horizontal scanner and vertical scanner were tilted orthogonally. Y-scanner seems to be placed in the same line between the two mirrors in the YZ plane (shown in Fig. 2(a)), while in fact it was at different height from the two mirrors, shown as in Fig 2(b). This led to a much compact design.

\section{BEAM DISPLACEMENTS}

The light was scanned on the retina in a raster pattern with a horizontal scanner and a vertical scanner (Cambridge Technology; 6220M40 galvanometric scanner, $\pm 20^{\circ}$ ). The two scanners were separated by a relay telescope designed to make them optically conjugate to each other and to the entrance pupil of the eye. This minimized the movement of the scanning beam at the pupil.
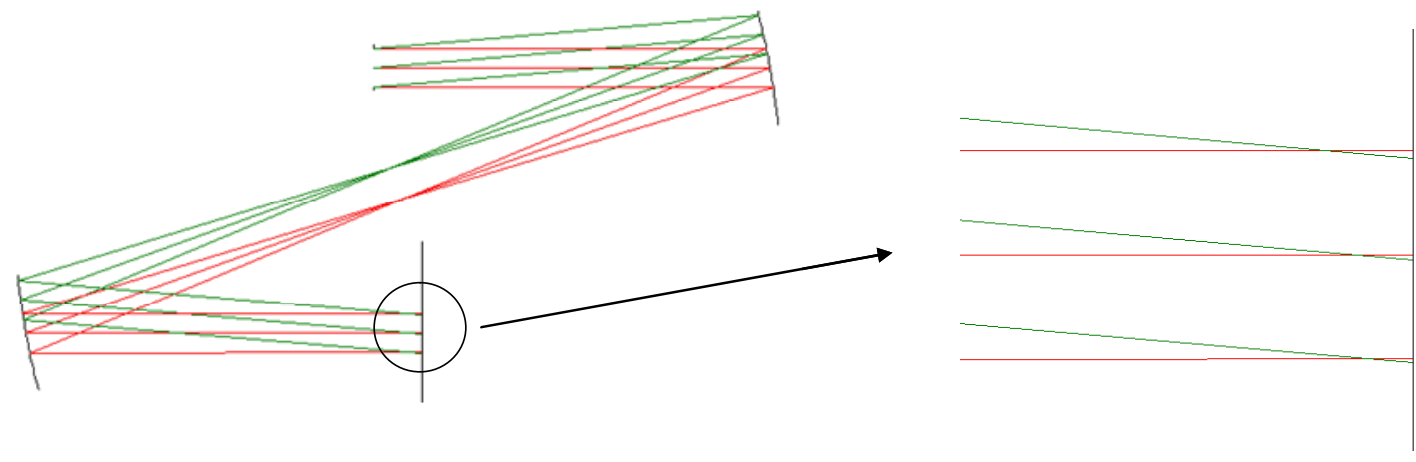

Fig. 3 Illustration of beam shift for different field angles in an afocal telescope. The right side is a magnified view of region near the image plane.

The chief rays from various field angles did not all pass through the center of the pupil. The beam was shifted as a function of the field angle as shown in Fig. 3. This would degrade the system performance in several ways. First, this resulted in beam clipping at the pupil, which caused intensity fluctuation as a function of scanning angle. Second, the phase correction the DM applied also depended on the scanning angle resulting in the AO system suffering a kind of anisoplanatism. Third, the speed of the scanners was much 
faster than the response time of the wavefront sensor and the DM. Hence, the ShackHartmann wavefront sensor measured the averaged wavefront and high spatial frequency aberrations may be averaged out and uncompensated.

Both horizontal and vertical scanners produce beam shifts. The shifts have to be minimized for the optimal performance. As a general rule, the shift needs to be less than half of the sub-aperture at the Shack-Hartman wavefront sensor.

\section{Design implementation:}

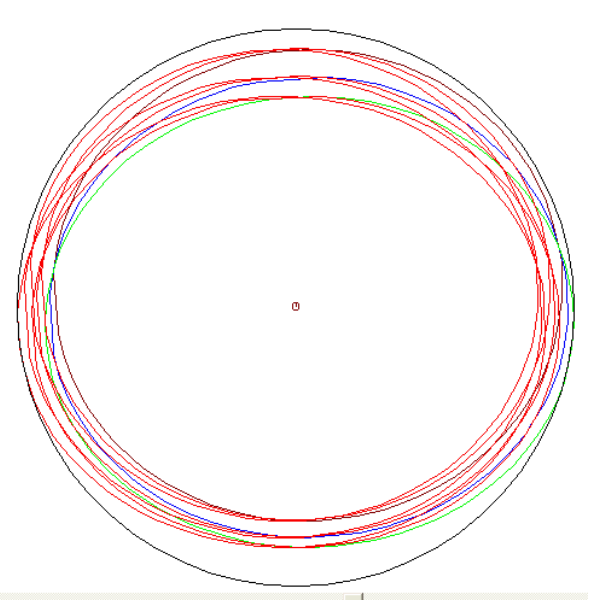

Fig. 4(a)

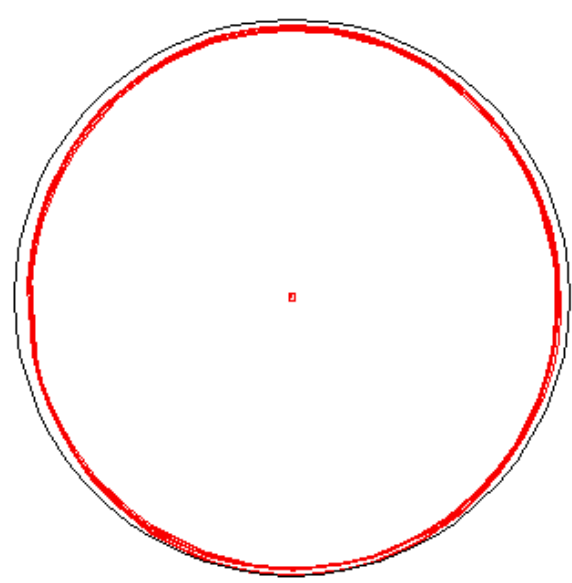

Fig. 4(b)

Fig. 4 illustration of the beam shifts of various scanning angles at the pupil plane of the eye of the AO-OCT design. (a) large beam shifts when mirrors are rotated in the same planes (b) small beam shift when mirrors are rotated orthogonally

The beam shift is proportional to the tilt angle of the reflective mirror and the F-number. So a smaller radius of curvature of the reflective mirror is preferred to minimize the beam displacement due to the scanning. This is beneficial for a compact design. However, a small radius of curvature of the mirrors would increase aberrations. Radii of curvatures were compromised to meet the design specification of both aberrations and beam displacements.

By rotating the second mirror orthogonally to the first mirror in the afocal telescope, both aberrations and beam displacements are reduced. Beam shifts of various scanning angles at the pupil plane of the eye of the AO-OCT design is illustrated in Fig.4. Beam shifts are large when mirrors are rotated in the same planes in the telescopes as shown in Fig. 4(a), while beam shifts are much smaller when mirrors are rotated orthogonally in the telescopes as shown in Fig. 4(b). 


\section{COMPENSATION OF SPECTACLE ABERRATIONS}

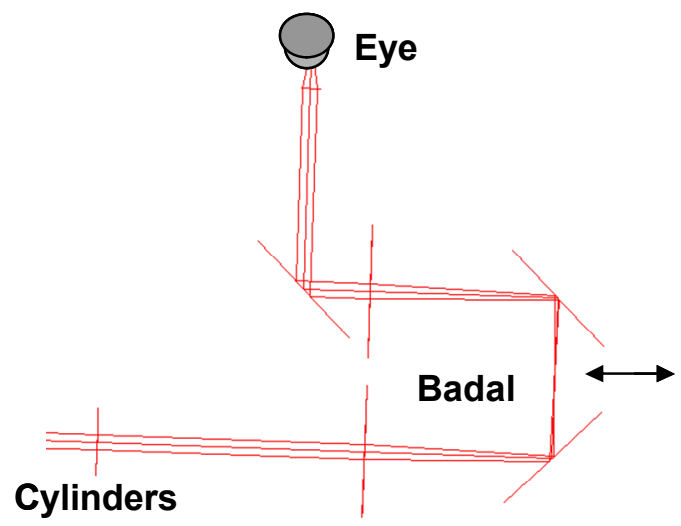

Fig. 5 Layout of optical apparatus for the spectacle aberration compensations. Defocus was compensated by the Badal optometer and astigmatism was compensated by the rotating cylinders in the eye.

We chose to use Badal lenses and rotating cylinders to compensate the large spectacle aberrations in the design of the AO-OCT for clinical use because of the limitations of the deformable mirrors. Current deformable mirror technology has limited strokes, and many of them have not been yet proven high reliable for long-term clinical testing.

The optical apparatus of the spectacle aberration compensations is shown in Fig. 5. The Badal optometers were used to compensate defocus. They were made of two achromatic transmissive lenses with focal lengths of $100 \mathrm{~mm}$ and two folding reflective mirrors. The two folding mirrors were controlled by one motorized translation stage. The amount of defocus the apparatus could compensate was linear to the moving distance of the two mirrors, as shown in Fig. 6. The two rotating cylinders were placed at the conjugate plane of the eye pupil to compensate the astigmatism in the eye.

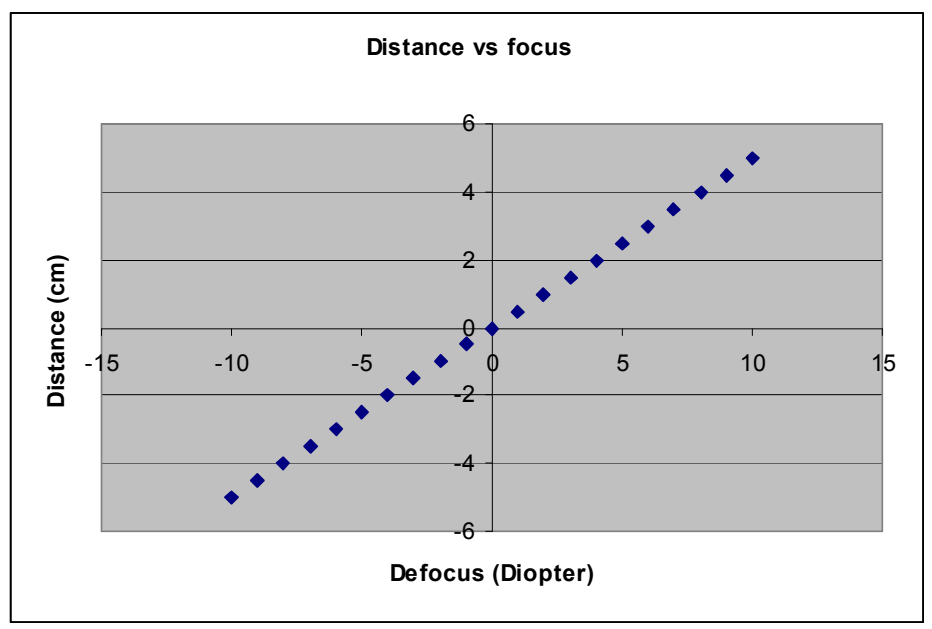

Fig.6. The amount of defocus compensated vs. the moving distance of the stage 


\section{OPTICAL SYSTEM}

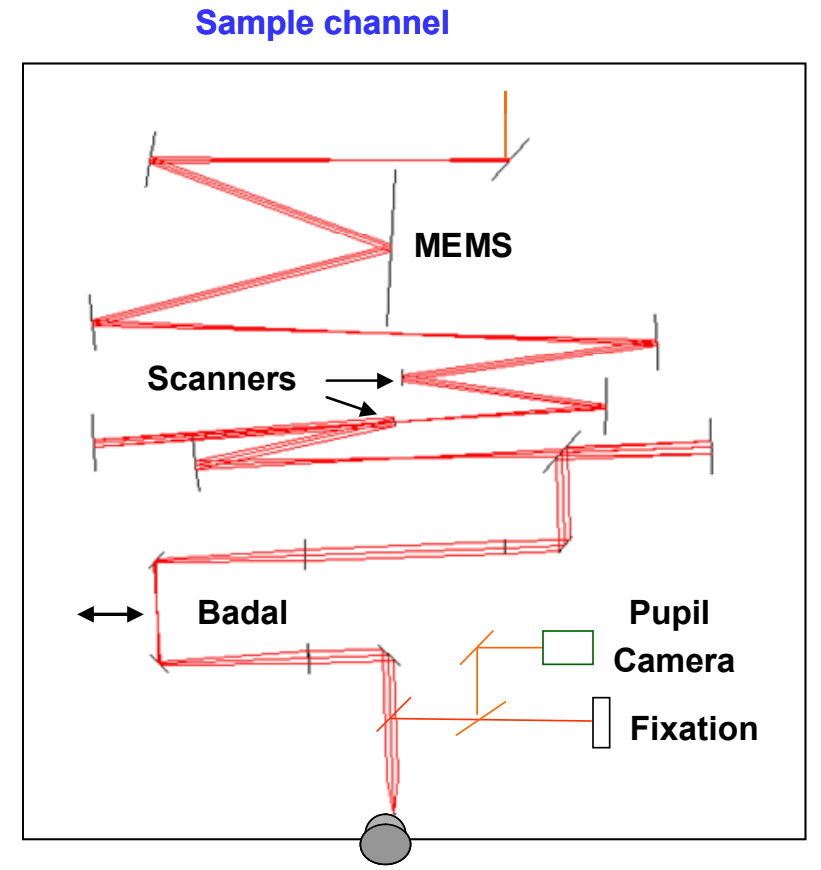

Fig. 7(a)

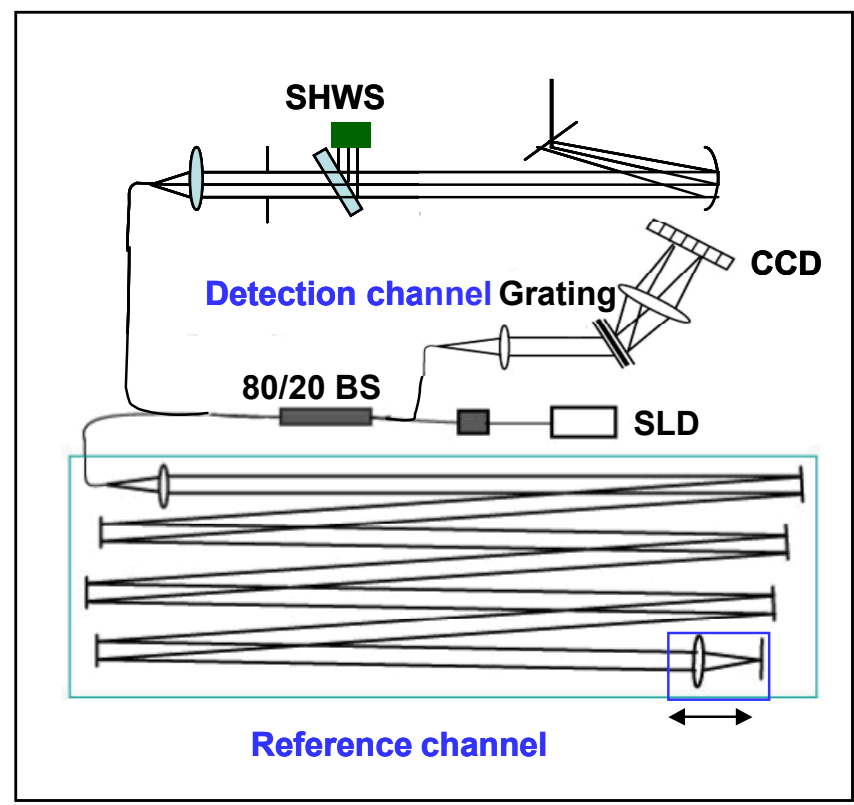

Fig. 7(b)

Fig. 7 AO-OCT optical system layout (a) Sample channel (b) reference channel and detection channel.

To make the AO-OCT system compact, the sample channel was set-up on one optical breadboard and the detection channel/reference channel were set up on the second optical breadboard, as shown in Fig. 7. The two breadboards were stacked together by supporting posts.

\section{SUMMARY}

In pursue of a compact AO-OCT system for clinical use, we chose to use Badal optometer and rotating cylinders to compensate defocus and astigmatism, and MEMS to compensate high-order aberrations. Different amount of spectacle aberration compensation was achieved by motorized stages and automated with the AO computer for ease of clinical use. In addition, the compact AO-OCT was optimized to have minimum system aberrations to reduce $\mathrm{AO}$ registration errors and improve $\mathrm{AO}$ performance by rotating mirrors orthogonally and reducing the rotation angles. 


\section{ACKNOWLEDGEMENT}

This research was supported by NIH grant EY014743 and performed under the auspices of the U.S. Department of Energy by Lawrence Livermore National Laboratory in part under contract W-7405-Eng-48 and in part under Contract DE-AC52-07NA27344.

\section{REFERENCES}

1. K. Thorn, J. Qu, R. Jonnal, and D. Miller, "Adaptive optics flood-illuminated camera for high speed retinal imaging," Invest. Ophthalmol. Vis. Sci. 44, 10021008 (2003).

2. A. Roorda, F. Romero-Borja, W. Donnelly, H. Queener, T. Herbert, and M. Campbell, "Adaptive optics scanning laser ophthalmoscopy," Opt. Express 10, 405-412 (2002).

3. B. Hermann, E.J. Fernandez, A. Unterhubner, H. Sattmann, A.F. Fercher, and W. Drexler, P.M. Prieto and P.Artal, "Adaptive-optics ultrahigh-resolution optical coherence tomography," Opt. Lett. 29, 2142-2144 (2004).

4. R. J. Zawadzki, S. M. Jones, S. S. Olivier, M. Zhao, B. A. Bower, J. A. Izatt, S. Choi, S. Laut, and J. S. Werner, "Adaptive-optics optical coherence tomography for high-resolution and high-speed 3D retinal in vivo imaging," Opt. Express 13, 8532-8546 (2005).

5. Y. Zhang, B. Cense, J. Rha, R. S. Jonnal, W. Gao, R. Zawadzki, J. Werner, S. Jones, S. Olivier, and D. T. Miller, "High-speed volumetric imaging of cone photoreceptors with adaptive optics spectral-domain optical coherence tomography," Opt. Express 14, 4380-4394 (2006).

6. W. Welford, Aberrations of optical systems, Adam Hilger, Bristol (1989). 